\title{
Investigating the influence of extraction methodology, and certain other experimental parameters on bacterial response to selected quorum-modulatory plant extracts \\ Pooja Patel, Chinmayi Joshi, Vijay Kothari* \\ Institute of Science, Nirma University, Ahmedabad-382481, India \\ *vijay.kothari@ nirmauni.ac.in
}

\begin{abstract}
In context of the global threat of antimicrobial resistance (AMR), a large number of labs throughout the world are investigating plant extracts for their possible anti-pathogenic efficacy. Outcome of protocols employed for screening the plant extracts for such activities are influenced by multiple factors. Among them, this study has found the method used for extraction, size and volume of the assay system, to be important factors. Further we demonstrate the extracts prepared by microwave- and vacuum-assisted extraction methods to be stable with respect to their anti-pathogenic efficacy over a long period of storage.
\end{abstract}

Keywords- Microwave Assisted Extraction (MAE); Vacuum Assisted Extraction (VAE); Caenorhabditis elegans; Quorum Sensing (QS)

\section{Introduction}

Medicinal plants are currently being actively investigated as a potential source of therapeutic phytochemicals that may lead to the development of novel drugs, cosmetics or nutraceuticals [1]. Biological activities of plant-based formulations are significantly dependent on extraction solvent and technique used. With a notable variety of methods present, selection of proper extraction method needs meticulous evaluation [1]. Further, same extract when tested for a particular biological activity using different protocols, results may vary. Present study attempted to investigate effect of certain important experimental factors on outcome of experiments aimed at assessing anti-virulence potential of plant extracts.

\section{Materials and Methods}

\section{A. Plant materials}

Peel of Punica granatum L. and Seeds of Phyllanthus emblica were procured (during 2015-16) from the fruits purchased from local market in the city of Ahmedabad and stored in air tight jar. They were authenticated for their unambiguous identity by Dr. Archana Mankad, Botany Dept., Gujarat University, Ahmedabad. Panchvalkal formulation (PentaphyteP- $5^{\circledR}$ ) was procured from Dr. Palep's Medical Research Foundation Pvt. Ltd., Mumbai.

\section{B. Test organisms}

Chromobacterium violaceum (MTCC 2656), were procured from MTCC (Microbial Type Culture Collection, Chandigarh). Pseudomonas aeruginosa was sourced from our internal culture collection. Nutrient broth and Pseudomonas broth (HiMedia, Mumbai) were used as growth media for C. violaceum and $P$. aeruginosa respectively. Incubation temperature and time for both these bacteria were kept at 37 ${ }^{\circ} \mathrm{C}$ for $22-24 \mathrm{~h}$.

\section{Extraction}

Dried peels and seeds were powdered to a uniform particle size. Sample to solvent ratio was kept constant for all the methods- $1 \mathrm{~g}$ seed powder in $50 \mathrm{ml}$ solvent. $50 \%$ ethanol (Ureca consumers co. op stores Ltd, Ahmedabad) was used as extraction solvent.

\section{Microwave assisted extraction (MAE)}

It was carried out in a microwave oven (Electrolux EM30EC90SS) at $720 \mathrm{~W}$ with intermittent cooling (each cooling cycle was of $40 \mathrm{~s}$ ) in $250 \mathrm{~mL}$ screw capped glass bottle (Borosil). Dark (brown) bottles 
were used to limit effect of light on plant material. Cap of the bottle was kept little loose during extraction. Total duration of microwave heating for extraction in ethanol was $70 \mathrm{~s}$.

E. Vacuum assisted extraction (VAE)

It was carried out at a working pressure of 7.36 psi. Total duration of extraction time was $15 \mathrm{~m}$ in at 65 ${ }^{\circ} \mathrm{C}$.

Extracts were clarified by centrifugation (Remi BZCI-8729) at 10,000 rpm for $15 \mathrm{~min}$, followed by filtration with Whatman \# 1 filter paper (Whatman International Ltd., England). After evaporation, dried extracts were reconstituted in DMSO (Merck, Mumbai). Extraction efficiency (Table 1) was calculated as percentage weight of the starting dried plant material. Reconstituted extracts were stored in autoclaved glass vials $\left(15 \mathrm{~mL}\right.$, Merck) under refrigeration $\left(4-8^{\circ} \mathrm{C}\right)$. Internal surface of the vial cap was covered with aluminium foil to prevent the leaching of any compounds from the cap into the extract [2].

Table 1. Extraction efficiency of extracts prepared by both methods

\begin{tabular}{|c|c|c|c|c|}
\hline & \multicolumn{2}{|c|}{ P. emblica } & \multicolumn{2}{c|}{ P. granatum } \\
\hline Method used for extraction & VAE & MAE & VAE & MAE \\
\hline Extraction efficiency (\%) & 10.30 & 12.40 & 35.30 & 28.20 \\
\hline Reconstitution efficiency (\%) & 84.46 & 88.71 & 93.48 & 94.32 \\
\hline $\begin{array}{c}\text { Final Concentration after } \\
\text { reconstitution (mg/mL) }\end{array}$ & 56.86 & 71.10 & 221.62 & 172.05 \\
\hline
\end{tabular}

\section{F. Broth dilution assay}

Effect of test extracts on bacterial growth and production of quorum sensing (QS)-regulated pigments were assessed using the methods described by us previously [3]. Appropriate vehicle control containing DMSO was also included in the experiment, along with abiotic control (containing extract, but no inoculum). Catechin was used as positive control.

G. In vivo assay

In vivo efficacy of the test extracts was evaluated using the nematode worm Caenorhabditis elegans as the model host through live-dead assay as detailed by us in Patel et al. [4]. Standard antibiotics- and catechin-treated bacterial suspensions were used as positive control.

\section{H. Statistical analysis}

All the experiments were performed in triplicate, and measurements are reported as mean \pm standard deviation (SD). Statistical significance of the data was evaluated by applying $t$-test using Microsoft Excel $^{\circledR} . p$ values less than 0.05 were considered to be statistically significant.

\section{RESULTS}

A. Choice of extraction method can have significant influence on anti-infective activity

$P$. granatum and $P$. emblica extracts prepared by MAE and VAE methods were assessed for their ability of influence bacterial growth and QS-regulated pigment production in vitro, and bacterial virulence towards $C$. elegans in vivo. Against $P$. aeruginosa both plant extracts prepared by any of the method had identical in vitro effect. However, in case of $P$. emblica, extract prepared by VAE could attenuate virulence of $P$. aeruginosa more than that prepared by MAE; whereas in case of $P$. granatum, extract prepared by MAE method scored better during in vivo assay (Figure 
1). Against $C$. violaceum, though the extracts prepared by both methods differed with respect to their in vitro effect, their in vivo efficacies were equivalent (Figure 2).

B. Vessel size and reaction volume are important determinants of experimental output

Effect of Panchvalkal formulation (PF) on C. violaceum and P. aeruginosa (Figure 3) was assessed in two different sized vessels: $10 \mathrm{~mL}$ test tubes (reaction volume $1 \mathrm{~mL}$ ), and $250 \mathrm{~mL}$ flask (reaction volume $100 \mathrm{~mL}$ ). PF could influence bacterial growth, catalase and haemolytic activity, and pigment production to a greater extent when experiments were performed in larger vessel size with higher reaction volume. Similar observation could be made while assessing effect of PF on $P$. aeruginosa catalase and haemolytic activity; however pyocyanin production was affected by PF more in tubes than in flasks.

C. Extracts prepared using MAE and VAE method retain their efficacy over long term $P$. granatum peel extract (PGPE) prepared by MAE, and P. emblica seed extract (PESE) prepared by VAE were first prepared and tested for their anti-pathogenic activity in May 2015. Then they were kept stored in refrigerator $\left(4-8{ }^{\circ} \mathrm{C}\right)$ and re-tested in Feb 2016 and Jan 2017 to investigate whether they still have retained their efficacy. In vitro and in vivo experiments (Figure 4) confirmed that these extracts were stable in terms of their activity against $C$. violaceum over a period of almost 19 months. 


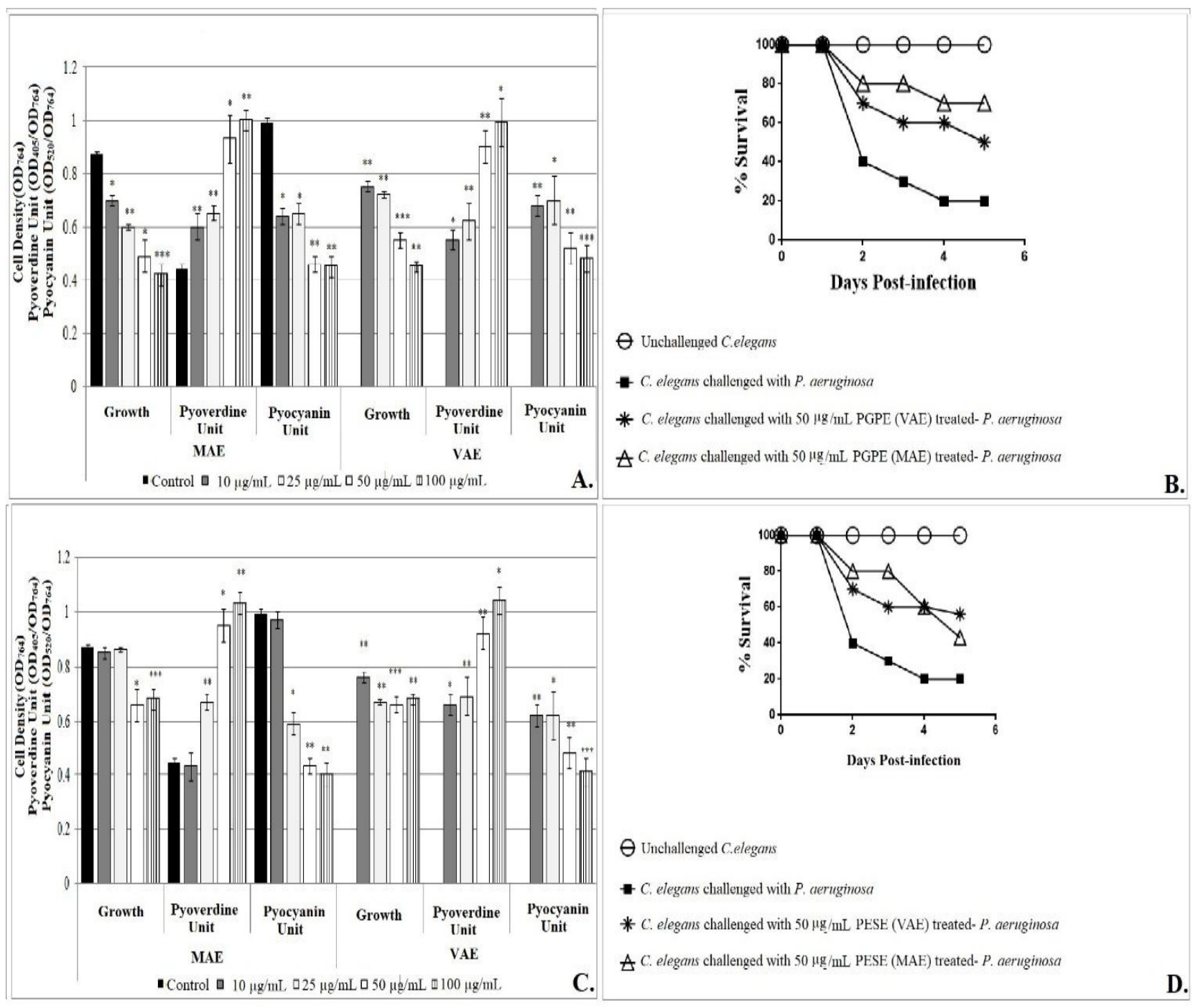

Figure 1: Comparison of in vitro and in vivo efficacy of plant extracts prepared by VAE and MAE against $P$. aeruginosa

(A) Effect of PGPE on growth and QS-regulated pigment production in $P$. aeruginosa (B) PGPE prepared by MAE scored better in vivo anti-virulence effect than that prepared by VAE (C) Effect of PESE on growth and QSregulated pigment production in $P$. aeruginosa (D) PESE prepared through both extraction methods were statistically at par with respect to their in vivo efficacy

During in vitro experiments, bacterial growth was measured as $\mathrm{OD}_{764}$; OD of pyoverdine was measured at $405 \mathrm{~nm}$, Pyocyanin was measured at $520 \mathrm{~nm}$. Pyoverdine Unit was calculated as the ratio $\mathrm{OD}_{405} / \mathrm{OD}_{764}$ (an indication of pyoverdine production per unit of growth); Pyocyanin Unit was calculated as the ratio $\mathrm{OD}_{520} / \mathrm{OD}_{764}$ (an indication of pyocyanin production per unit of growth); Catechin $(50 \mu \mathrm{g} / \mathrm{mL})$ inhibited pyoverdine $17.13 \% * * \pm 0.06$ and pyocyanin $23.65 \% * \pm 0.04$ production without affecting the bacterial growth.

During in vivo experiments, DMSO present in the 'vehicle control' at $0.5 \% \mathrm{v} / \mathrm{v}$ did not affect virulence of the bacterium towards $C$. elegans. DMSO $(0.5 \% \mathrm{v} / \mathrm{v})$ and plant extracts at tested concentrations showed no toxicity towards the worm. 

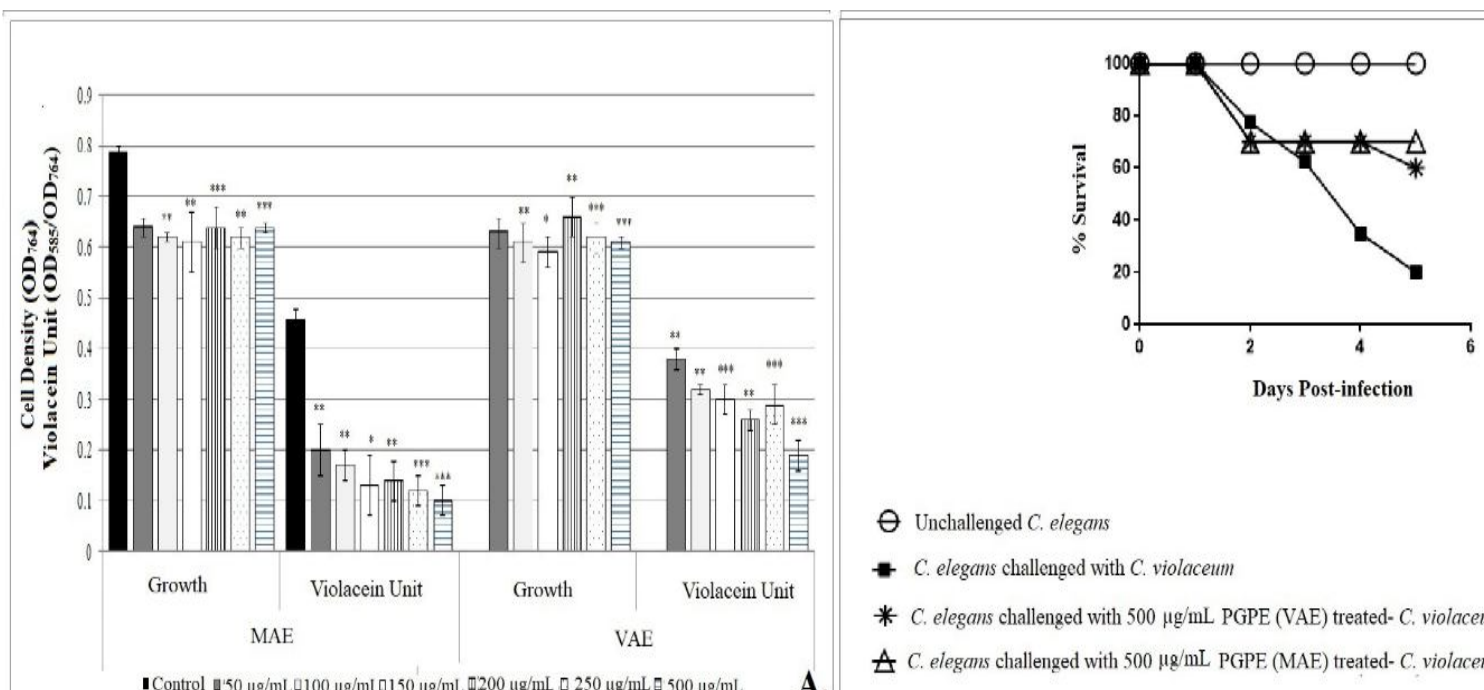

$\theta$ Unchallenged $C$, elegans

* C. elegans challenged with $C$. violaceum

* C. elegans challenged with $500 \mu \mathrm{g} / \mathrm{mL}$ PGPE (VAE) treated-C. violaceum

A.

A C. elegans challenged with $500 \mu \mathrm{g} / \mathrm{mL}$ PGPE (MAE) treated-C. violaceum

B.
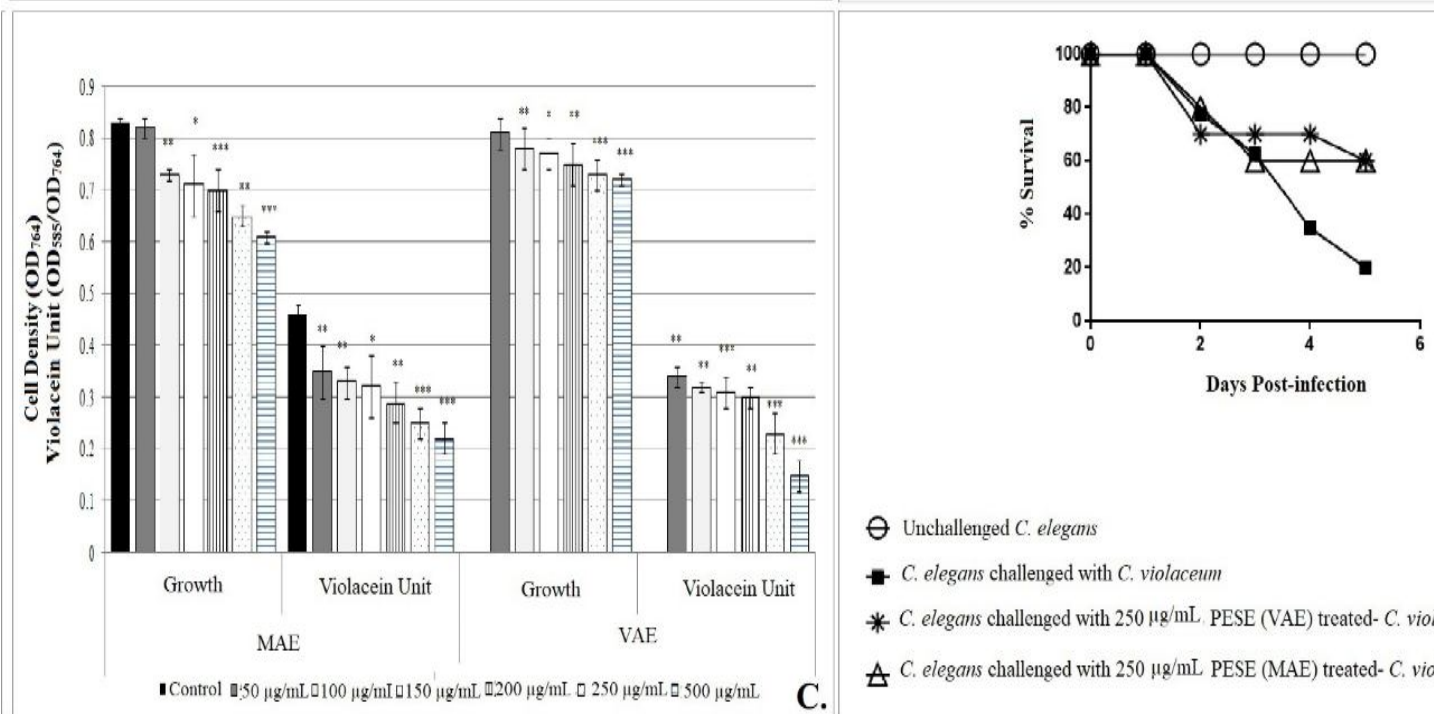

$\theta$ Unchallenged $C$. elegans

- C. elegans challenged with $C$. violaceum

* C. elegans challenged with $250 \mu \mathrm{\mu g} / \mathrm{mL}$. PESE (VAE) treated- C. violaceum

C.

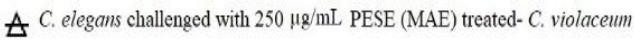

D.

Figure 2: Comparison of in vitro and in vivo efficacy of plant extracts prepared by VAE and MAE against $C$. violaceum

(A) Effect of PGPE on growth and QS-regulated pigment production in C. violaceum (B) PGPE prepared through both extraction methods were statistically at par with respect to their in vivo efficacy (C) Effect of PESE on growth and QS-regulated pigment production in $C$. violaceum (D) PESE prepared through both extraction methods were statistically at par with respect to their in vivo efficacy

During in vitro experiments, Bacterial growth was measured asOD ${ }_{764}$; OD of violacein was measured at $585 \mathrm{~nm}$, and Violacein Unit was calculated as the ratio $\mathrm{OD}_{585} / \mathrm{OD}_{764}$ (an indication of violacein production per unit of growth); Catechin $(50 \mu \mathrm{g} / \mathrm{mL}$ ) did not exerted any effect on growth of $C$. violaceum, and inhibited violacein production by $47.69 \%^{* * *} \pm 0.03$.

During in vivo experiments, DMSO present in the 'vehicle control' at $0.5 \% \mathrm{v} / \mathrm{v}$ did not affect virulence of the bacterium towards C. elegans. DMSO $(0.5 \% \mathrm{v} / \mathrm{v})$ and plant extracts at tested concentrations showed no toxicity towards the worm. 

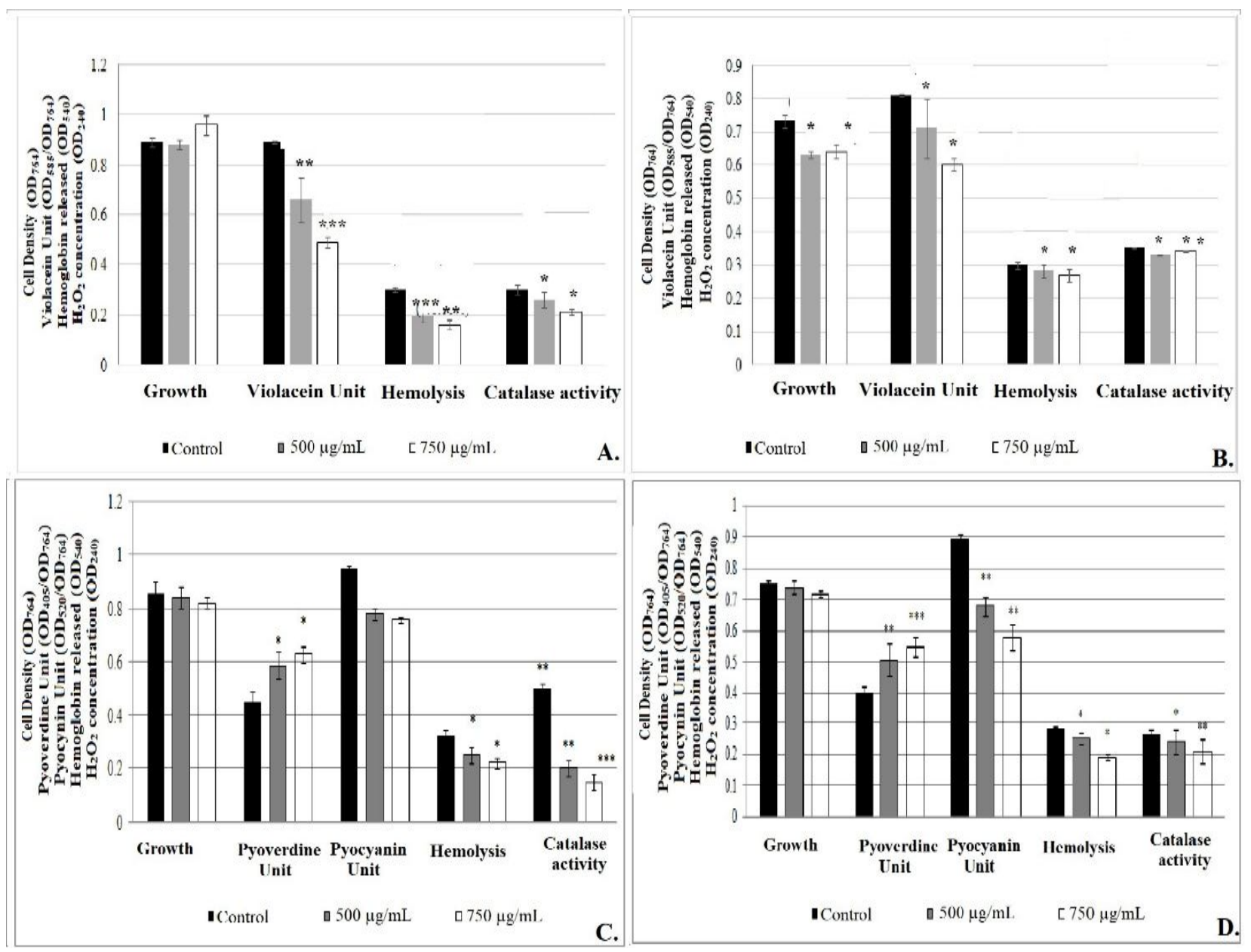

Figure 3: Influence of vessel size and reaction volume on PGPE's effect on various traits of $C$. violaceum and $P$. aeruginosa

Catalase assay was done by monitoring disappearance of $\mathrm{H}_{2} \mathrm{O}_{2}$ at $240 \mathrm{~nm}$; Hemoglobin concentration was measured at $\mathrm{OD}_{540}$.

Effect of PGPE on $C$. violaceum grown in (A) flask, and (B) test tube

Bacterial growth was measured asOD 764 ; OD of violacein was measured at $585 \mathrm{~nm}$, and Violacein Unit was calculated as the ratio $\mathrm{OD}_{585} / \mathrm{OD}_{764}$ (an indication of violacein production per unit of growth); Catechin $(50 \mu \mathrm{g} / \mathrm{mL}$ )

did not exerted any effect on growth of $C$. violaceum, and inhibited violacein

Effect of PGPE on $P$. aeruginosa grown in (C) flask and (D) test tube

Bacterial growth was measured as $\mathrm{OD}_{764}$; OD of pyoverdine was measured at $405 \mathrm{~nm}$, Pyocyanin was measured at $520 \mathrm{~nm}$. Pyoverdine Unit was calculated as the ratio $\mathrm{OD}_{405} / \mathrm{OD}_{764}$ (an indication of pyoverdine production per unit of growth); Pyocyanin Unit was calculated as the ratio $\mathrm{OD}_{520} / \mathrm{OD}_{764}$ (an indication of pyocyanin production per unit of growth); Catechin $(50 \mu \mathrm{g} / \mathrm{mL})$ inhibited pyoverdine $17.13 \% * * \pm 0.06$ and pyocyanin $23.65 \% * \pm 0.04$ production without affecting the bacterial growth. 


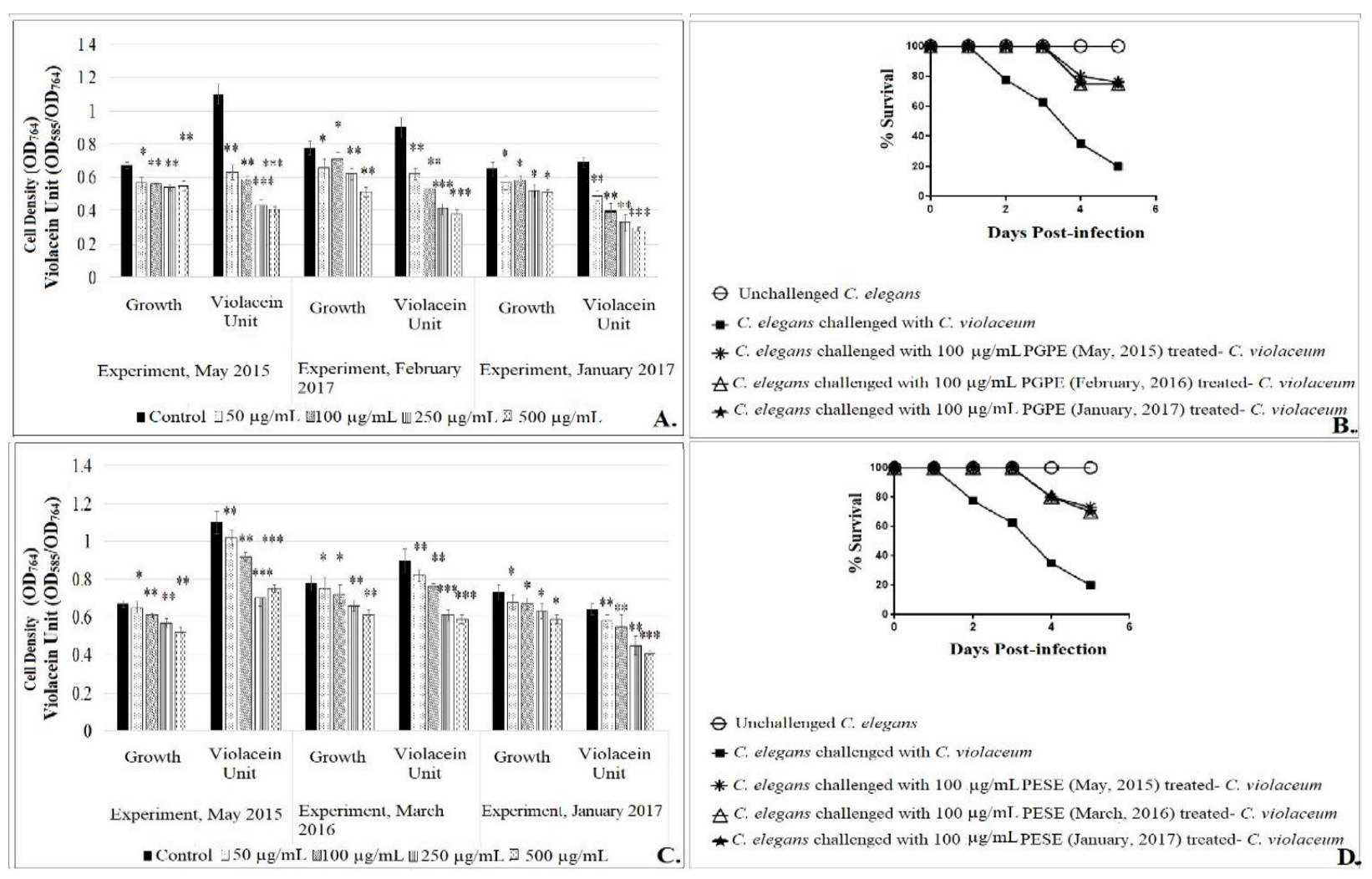

Figure 4: Extracts prepared by VAE and MAE retain their anti-pathogenic effect over a long period of refrigerated storage

A-B. Results for PGPE; C-D. Results for PESE

\section{Conclusion}

Currently plant sources are being actively investigated for a variety of biological and pharmacological activities in search of novel leads of pharmaceutical relevance. However owing to a variety of screening protocols being used for preliminary assessment of desired biological activity, direct comparison between results of different protocols remains tricky. This study has indicated that due attention should be paid to factors like choice of extraction method, vessel size, reaction volume, etc. while fixing the screening strategy. This study has also found the extracts prepared by MAE and VAE method to be stable over a refrigerated storage of over a year. Owing to their inherent character of offering fast heating (MAE), and boiling at lower temp (VAE), these methods are also considered suitable for extraction of heat-labile phytocompounds. Such studies with more varieties of extraction methods, on other experimental determinants, and additional pathogenic bacteria will provide better insight enabling the researchers to adopt potentially more reliable and reproducible screening protocols.

\section{ACKNOWLEDGMENT}

Authors thank Nirma Education and Research Foundation (NERF, Ahmedabad) for financial and infrastructural support 


\section{REFERENCES}

[1] N. N. Azwanida, "A Review on the Extraction Methods Use in Medicinal Plants, Principle, Strength and Limitation", Medicinal Aromatic Plants, 04(03), 2015. Available at: http://dx.doi.org/10.4172/2167-0412.1000196.

[2] P. Houghton, A. Raman, "Laboratory Handbook for the Fractionation of Natural Extracts", Chapman and Hall:London, UK; 18, 1988

[3] Joshi, V. Kothari, and P. Patel, "Importance of selecting appropriate wavelength, while quantifying growth and production of quorum sensing regulated pigments in bacteria", Recent Patents Biotechnol, 2016; 10(2), PP.145-152, 2016. Available at: http://dx.doi.org/10.2174/1872208310666160414102848.

[4] P. Patel, C. Joshi, H. Palep, and V. Kothari, "Anti-infective potential of a quorum modulatory polyherbal extract (Panchvalkal) against certain pathogenic bacteria", J Ayurveda Integr Med, 2018. Available at: https://doi.org/10.1016/j.jaim.2017.10.012 